\title{
Teuscher Activator- Old But Still Effective
}

\author{
Harshil Naresh Joshi ${ }^{*}$, Santosh Kumar Goje ${ }^{2}$, Jay Sureshkumar Soni ${ }^{3}$, \\ Rameshwar H Taluja ${ }^{4}$, Swetha Taluja ${ }^{5}$ and Manjot Kaur ${ }^{6}$ \\ ${ }^{1,2}$ K. M. Shah Dental College and hospital, Sumandeep Vidyapeeth, \\ Pipariya Waghodia, vadodara, Gujarat India \\ ${ }^{3}$ Ahmedabad Dental College \& Hospital, Ahmedabad, Gujarat, India \\ ${ }^{4} B D S, M D S$ (Prosthodontist and Implantologist) \\ ${ }^{5} B D S$, PGCOI( Dental Surgon) \\ ${ }^{6}$ BDS Baba Jaswant Singh Dental College \&t Hospital, Punjab, India
}

\section{ABSTRACT}

Skeletal Class II malocclusion with mandibular retrognathism is one of the most common problems in orthodontic practice. There are assorted treatments to correct them, such as Myo functional appliances \&t Fixed Functional appliances. Functional appliances are commonly used for the treatment of class II malocclusions with growing individuals. The accomplishment of treatment with a functional appliance depends on the patient's cooperation and skeletal growth status. Severe discrepancy cases need to address step via step vise advancement. This case report discusses the importance of two-step advancement for a more skeletal result. Anderson's activator with torquing spurs was used. After the active phase of two-step functional therapy. The Rickanator was used for retention therapy followed by fixed mechanotherapy. The activator successfully corrects the problem of the retrusive mandible \&t proclined anterior with favorable mandibular growth \&t restraining maxillary anterior. The active treatment lasted for 22 months. This two-phase treatment yielded a pleasing profile and good occlusion in this patient.

\section{KEY WORDS: MANDIBULAR RETROGNATHISM, FIXED MECHANOTHERAPY, RETRUSIVE MANDIBLE \& PROCLINED} ANTERIOR.

\section{INTRODUCTION}

- Class II malocclusion is considered as one of the most prevalent types of malocclusion encountered in routine orthodontic practice and described by an improper relationship among the upper and lower jaws caused by dental or skeletal problems or a combination of both in growing patients (McNamara, 1981).

- According to McNamara, (1981) Class II malocclusions result mostly from a relative mandibular retrognathism rather than from maxillary prognathism. Hence, any appliance with the ability to stimulate significant mandibular growth would play a pivotal role in the clinician's treatment planning.

- Two-Step Advancement for severe skeletal

Biosc Biotech Res Comm P-ISSN: 0974-6455 E-ISSN: 2321-4007

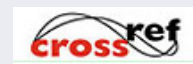

Identifiers and Pagination

Year: 2021 Vol: 14 No (6) Special Issue

Pages: 20-24

This is an open access article under Creative

Commons License Attribn 4.0 Intl (CC-BY). DOI: $h t t p: / / d x$.doi.org/10.21786/bbrc/14.6.4 malocclusions class II, which includes growth modification with functional appliances followed by fixed mechanotherapy has been advocated as an appropriate treatment approach (Bowman, 1988).

- Correction of mandibular deficiency in a skeletal Class II patient poses a great challenge.

- Management of Class II malocclusion using functional appliances has become popular since the introduction of activator by Andersen. Teuscher4 activator is a variety of functional orthopedic appliances. Their actions depended on holding the mandible in an advanced position and create muscle stretching. To inhibit the forward growth of the maxilla, stimulate condylar growth and improvement of the muscle pattern (Teuscher, 1978).

- Diagnosis of the skeletal deformity plays a pivotal role in the final outcome. This case report describes the functional jaw orthopedic treatment of a dentoalveolar maxillary component growing male with mandibular deficiency. A modified Activator was used for skeletal correction which was followed by fixed mechanotherapy.

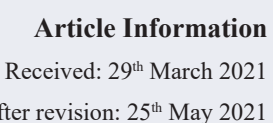

ccepted after revision: $25^{\text {th }}$ May 2021 
History, clinical findings, and diagnosis: The present case report is about a 10-year 4 month-old male patient who reported to the dept. Of Orthodontics, K. M. Shah Dental College \&t Hospital with the complaint of protrusion of upper teeth. Diagnostic Examination of the case was performed using a standardized protocol of the Department As followed. Extra-oral examination revealed facial convexity with apparent mandibular deficiency and incompetent lips. Intraoral examination revealed the presence of mixed dentition with class I molar relationship and increased overjet $(12 \mathrm{~mm})$ with 100\% deep bite. (Fig 1.a to 1.e) The extra oral examinations reviles the lower facial third was reduced indicating a horizontal growth pattern.(Fig 2.a to 2.c) The upper facial midline was coinciding with the upper dental midline \& the Lower dental midline was shifted by $2 \mathrm{~mm}$ to the right side. (Table. 1).

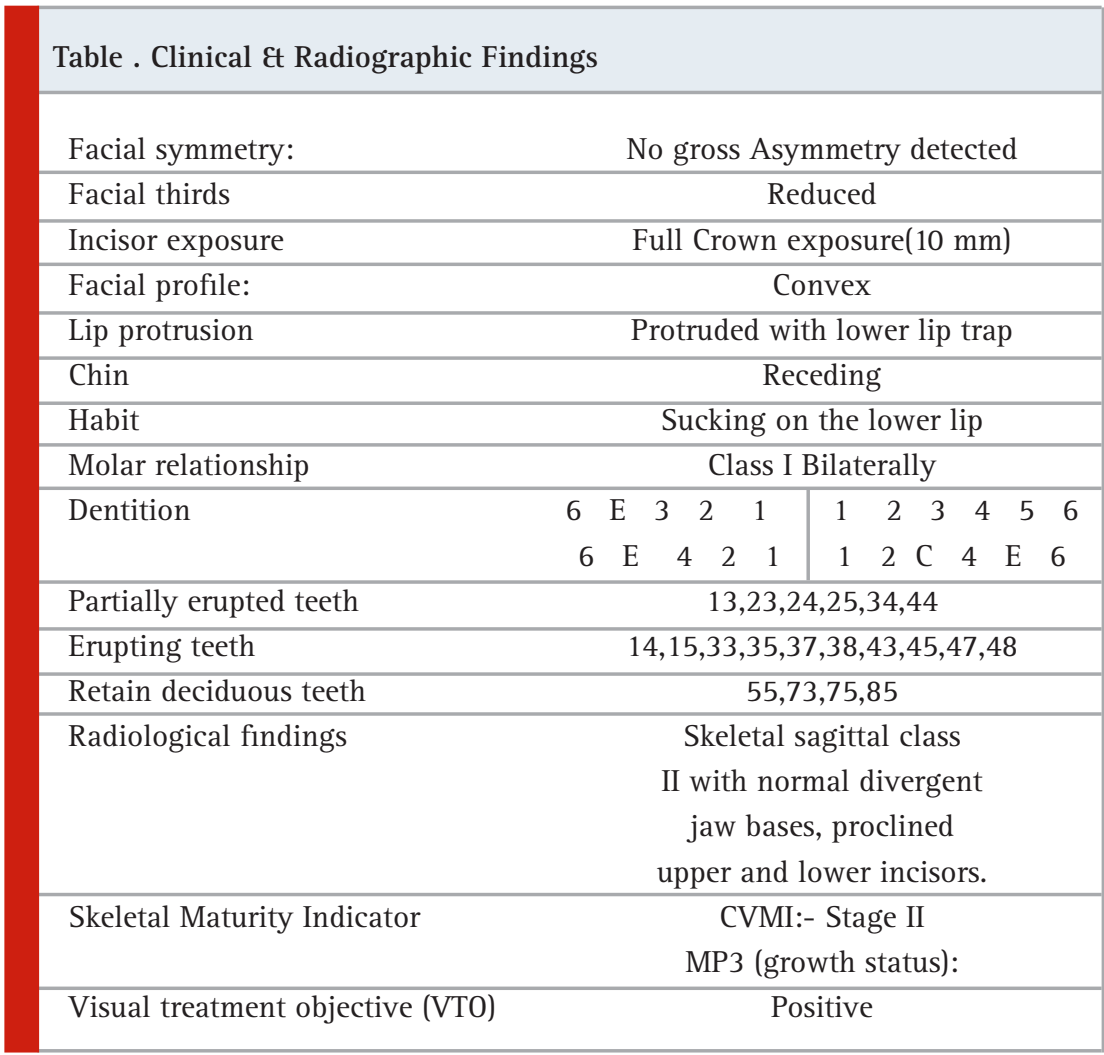

The reason behind the dental class I molar relations could be the premature loss of deciduous tooth which would have lead to the mesial migration of mandibular molars. Based on cephalometric measurements,(Fig 3) the patient was diagnosed as a case of skeletal Class II malocclusion with a horizontal -growth pattern. The question for appliance selection was Activator with headgear or activator alone (With Modification) versus twin block. An Activator with headgear could be the first choice as it corrects dentoalveolar proclination of maxilla \&t favorable advancement in mandible. The selective trimming of the activator would help the operator to distalize mandibular posterior teeth for maintaining the class 1 molar on both sides along with creating eruptive space for a mandibular right canine. However, the headgear effect of the activator alone is also well known \&t documented.

The Other concern of the parent's being reluctant for any extraoral appliance visible outside the oral cavity. That could be because the other Children might tease her or Injury during regular wearing \& removing or fight during play. The parents were traveling more than $100 \mathrm{~km}$ for this treatment so the approach towards the treatment planning was minimalistic. To avoid any unwanted visit by the patient. Considering the situation Activator appliance with modification for dentoalveolar proclination was decided. The appliance was easy to use, sturdy enough not to break easily to wear \&t remove \&t easy to clean.

The Other concern of the parent's being reluctant for any extraoral appliance visible outside the oral cavity. That could be because the other Children might tease her or Injury during regular wearing \& removing or fight during play. The parents were traveling more than $100 \mathrm{~km}$ for this treatment so the approach towards the treatment planning was minimalistic. To avoid any unwanted visit by the patient. Considering the situation Activator appliance with modification for dentoalveolar proclination was decided. The appliance was easy to use, sturdy enough not to break easily to wear \&t remove \&t easy to clean (Varlik et al., 2008).

The present case typically shows the importance of soft tissue over skeletal relationship \&t dental occlusion. The choice of appliance that is "Activator with Torquing spurs" also called "Teuscher Activator"(Fig. 4) has an 
excellent for efficacy to control the tooth position.6 Selective trimming can move the tooth to a favorable position, \& distalization was required to maintain the molar relationship \&t to create the space for the mandibular right canine. Our treatment objectives were to boost the mandibular growth $\mathrm{Et}$ restrict the dentoalveolar proclination of the maxilla. As the overjet was more two-step advancement was planned.

Appliance Design and Mechanics: The activator was constructed by observing the functional analysis and facial vertical measurements. The skeletal advancement was planned in two steps. For the 1st step, the construction bite was recorded with a vertical height of $5 \mathrm{~mm}$ in the premolar region and an advancement of $5 \mathrm{~mm}$. The 5 $\mathrm{mm}$ advancement of the mandible resulted in a superclass I molar relation. As the mandibular midline shift was laterocclusion rather than laterognathia, correction of it was panned at the time of fixed mechanotherapy. The design of the Teuscher Activator was taken as a reference. The Teuscher activator is a quite simple still robust acrylic-based activator. The design of the appliance with torquing spurs on the upper incisors can be adapted to the patient's needs. Incisal capping was done in mandibular anterior to prevent proclination.

The four torquing spurs (one on each maxillary incisor) were fabricated of $0.9-\mathrm{mm}$ resilient SS (stainless steel) wire and were well embedded in the wax bite between the lower and upper incisors. The spurs made contact with the maxillary incisors below the gingival margin (Cervical 1/3). To obtain better torque on maxillary incisors during the headgear effect the spurs are the most useful auxiliary. The torquing spurs prevented palatal tipping of the crowns. All the necessary Basic appliance care instructions were explained to the patient and parent both. The patient was asked to use the appliance as much as he could.

As patient's school timing was around 8-10 hours a day. So the expected wearing time was around 14 hours a day. On the day of the holiday, the patient was asked to wear it a full day. The first two appointments are planned at the interval of 2 weeks to check the comfort and compliance of the patient. The patient was quite cooperative with all the instructions $\mathrm{Ct}$ with the appliance use. The routine visits were planned at the interval of 6-8 weeks for selective grinding of the acrylic framework for redirecting the erupting teeth.

Follow up: The patient was wearing the appliance nicely Et oral hygiene was also satisfactory. Selective trimming of the acrylic framework was performed on every visit for distalization of mandibular molar \& eruption of posteriors for correction of deep bite. As the Patient is a horizontal grower the correction of the deep bite was done with the eruption of mandibular posterior teeth. The torque spurs were readapted as the maxillary anterior teeth' positions were changed. The distalization of the mandibular posterior leads to space opening for blocked mandibular right canine.(Fig 5.a to 6.c).
After 6 months of phase I, step two advancement was panned. The new construction bite was recorded in the advanced position; the patient showed an improved facial profile and bilateral super Class I molar and canine relationship. The excessive curve of spee of the lower arch was also leveled by the selective trimming. The advancement was stopped after achieving a straight profile. As the aim of myo-functional therapy was achieved, the patient was strapped up with a 0.022-inch slot; metal brackets; Pre adjusted Edgewise Appliance with MBT mechano-therapy (3M Unitek, Monrovia, California). (Fig 7.a to 8.c)The fixed mechanotherapy aimed to achieve normal overjet, overbite, no rotation with ideal interdigitation.

Figure 1: Pre Treatment Intra Oral Photographs

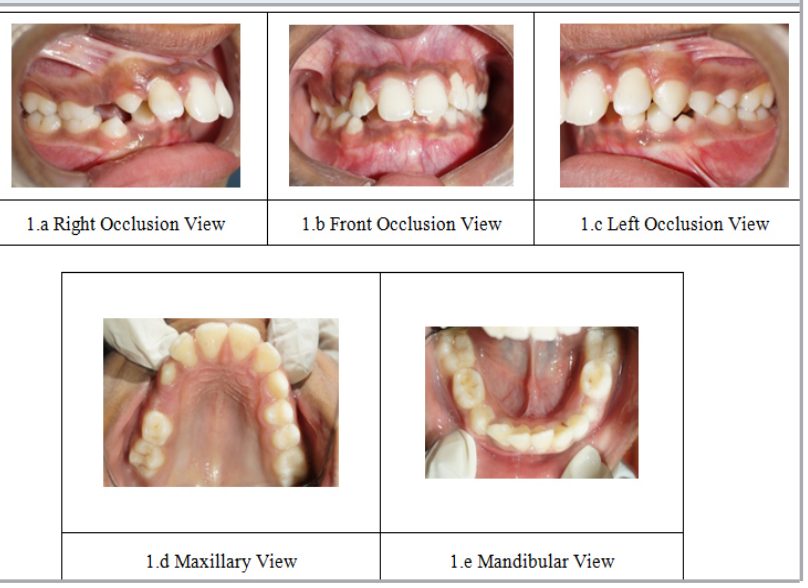

Figure 2: Pre Treatment Extra Oral Photographs

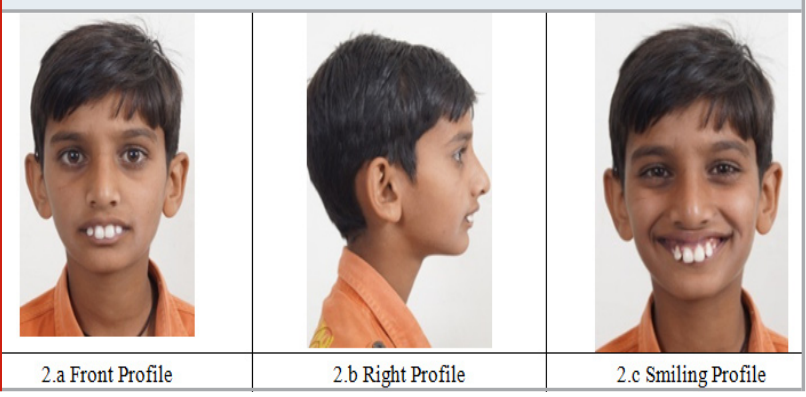

Figure 3: Lateral Cephalogram

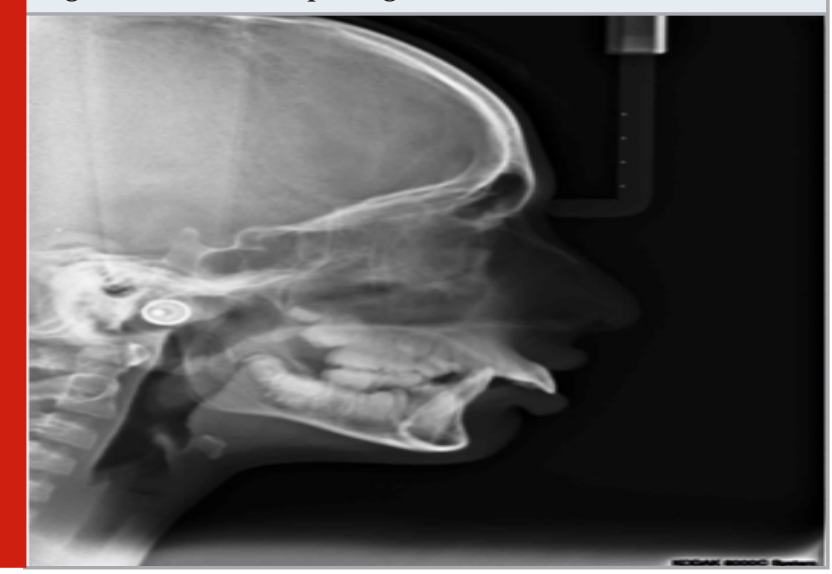


Figure 4: Right Occlusion View with Teuscher Activator Appliance design

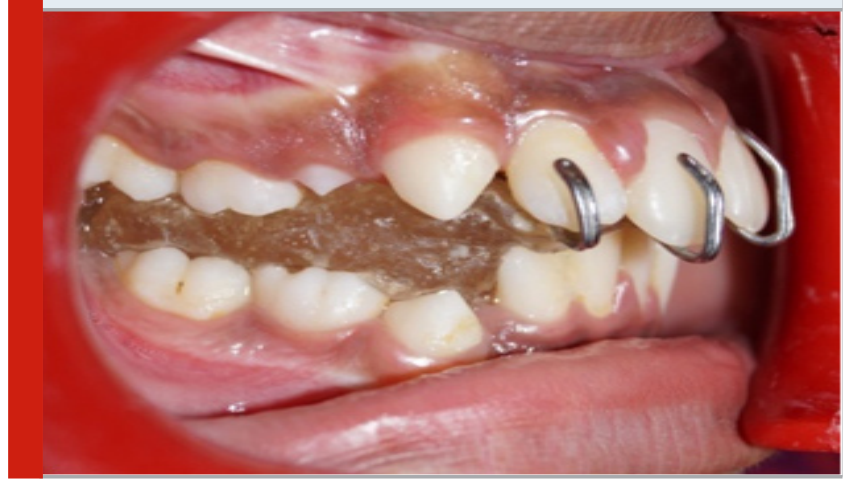

Table 2. Pre- Post Treatment Cephalometric measuments

Cephalometric values for the patient

Cephalometric variable Pre-treatment Post Functional

\begin{tabular}{|l|c|c|}
\hline SNA & $80^{\circ}$ & $81^{\circ}$ \\
\hline SNB & $70^{\circ}$ & $77^{\circ}$ \\
\hline ANB & $10^{\circ}$ & $4^{\circ}$ \\
\hline A1-B1(FH) & $12^{\circ}$ & $4^{\circ}$ \\
\hline U1-SN & $114^{\circ}$ & $106^{\circ}$ \\
\hline L1-NB & $25^{\circ}$ & $28^{\circ}$ \\
\hline U1-A-Pog & $45^{\circ}$ & $26^{\circ}$ \\
\hline L1-N-Pog & $14 \mathrm{~mm}$ & 5 \\
\hline SUBNASALE I & $-14 \mathrm{~mm}$ & -4 \\
T0 CHIN & & \\
\hline Sn-GoGn & $32^{\circ}$ & $34^{\circ}$ \\
\hline FMA & $22^{\circ}$ & $26^{\circ}$ \\
\hline
\end{tabular}

Figure 5: Intraoral Photograph after First step of Advancement
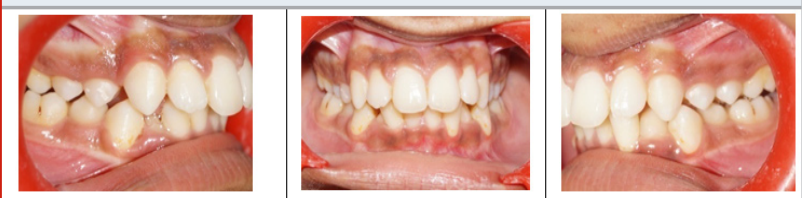

5 a Right Occlusion View

5.b Front Occlusion View

5.c Left Occlusion View

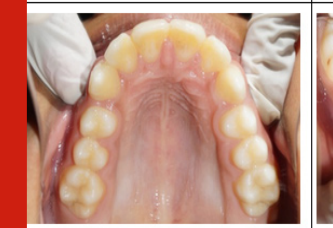

5.d Maxillary View

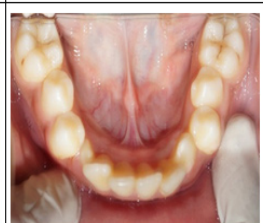

5.e Mandibular View

Retention: After the Myofunctional therapy, a Fixed inclined plane (Fig 7.d) was used for retention. It allows starting the fix mechanotherapy as it doesn't interfere with tooth movement. Wrap around retainer with anterior bite plate in maxillary arch $\&$ fixed lingual retainer was done in the mandibular arch.
Figure 6: Extraoral Photograph after First step of Advancement

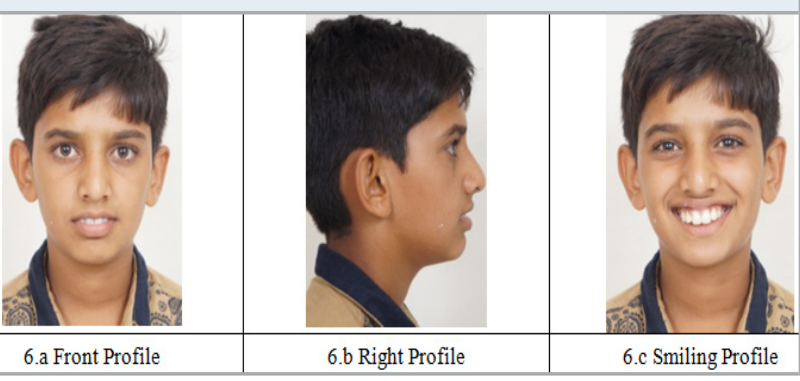

Figure 7: Intraoral Photograph after Fixed mechanotherapy

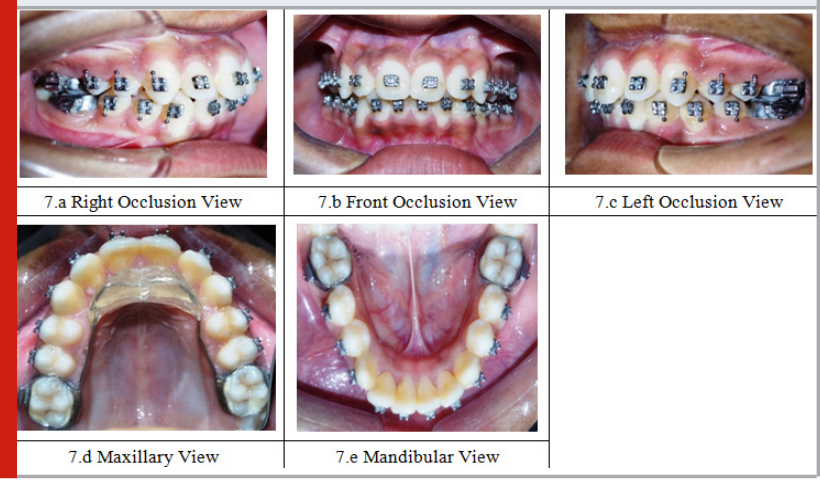

Figure 8: Extraoral Photograph after Fixed mechanotherapy

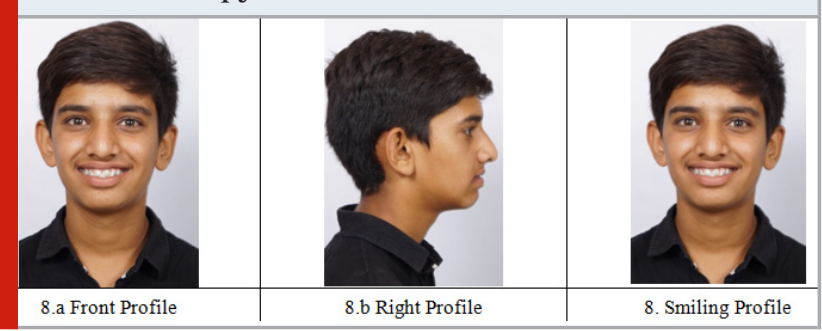

\section{DISCUSSION}

Many researchers have revealed the influences of activator on skeletal \&t dentoalveolar components (Woodside, 1973; Basciftci Faruk Ayhan et al., 2003; Ahlgren and Laurin, 1976). The activator stimulates condylar and as a result, the mandible grows. An influence on glenoid fossa remodeling has also been reported by many workers (Jakobsson, 1967; Hashim, 1991). Activator is designed to enhance the forward growth of the mandible by creating a functional displacement of the mandibular condyles downward and forward in the glenoid fossa (Hashim, 1991). Adaptive remodeling occurs on both articular surfaces of the temporomandibular joint to improve the maxilla-mandibular jaw relationship (Marschner and Harris, 1966; Ruf et al., 2001).

The activator and other tooth bone myofunctional appliances provide a maximum contact area with the mandibular jaw thus are more effective in holding the 
mandible forward constantly. Upper incisors in our patient showed some amount of retrusion which was similar to the findings of Tumer \& Gultan (1999). The modification done in the activator effectively maintains the torque during dentoalveolar correction of the maxillary arch. The incisal capping in lower incisors had prevented proclination. The functional therapy phase took 14 months which was longer than usual. As the two-step advancement was performed. The other reason for a long time was the distalization process \&t allowing time for the permanent teeth to erupt. The total duration of the treatment was 22 months. The patient and her parents were satisfied with the treatment result.

\section{CONCLUSION}

This case report presents a successful treatment of a class II division 1 case using two-step advancement therapy with the help of a modified activator. A pleasing well balanced orthognathic profile was achieved with the correction of all skeletal, dental \&t soft tissue problems.

\section{REFERENCES}

Ahlgren, J. and Laurin, C., 1976. Late results of activator-treatment: a cephalometric study. British Journal of Orthodontics, 3(3), pp.181-187.

Basciftci, F.A., Uysal, T., Büyükerkmen, A. and Sari, Z., 2003. The effects of activator treatment on the craniofacial structures of Class II division 1 patients. The European Journal of Orthodontics, 25(1), pp.87-93.

Basciftci, F.A., Uysal, T., Büyükerkmen, A. and Sari, Z., 2003. The effects of activator treatment on the craniofacial structures of Class II division 1 patients. The European Journal of Orthodontics, 25(1), pp.87-93.

Bowman, S.J., 1998. One-stage versus two-stage treatment: are two really necessary?. American journal of orthodontics and dentofacial orthopedics, 113(1), pp.111-116.

Hashim, H.A. 1991. Analysis of activator treatment changes. Aust Orthod J.12:100-4.
Jakobsson, S.O., 1967. Cephalometric evaluation of treatment effect on Class II, Division 1 malocclusions. American Journal of Orthodontics, 53(6), pp.446-457. Latchoumi, T.P., Ezhilarasi, T.P. and Balamurugan, K., 2019. Bio-inspired weighed quantum particle swarm optimization and smooth support vector machine ensembles for identification of abnormalities in medical data. SN Applied Sciences, 1(10), pp.1-10.

Marschner, J.F. and Harris, J.E., 1966. Mandibular growth and Class II treatment. The Angle Orthodontist, 36(1), pp.89-93.

McNamara, J.A., 1981. Components of Class II malocclusion in children 8-10 years of age. The Angle Orthodontist, 51(3), pp.177-202.

Ruf, S., Baltromejus, S. and Pancherz, H., 2001. Effective condylar growth and chin position changes in activator treatment: a cephalometric roentgenographic study. The Angle Orthodontist, 71(1), pp.4-11.

Singh, G.D. and Thind, B.S., 2003. Effects of the headgear-activator Teuscher appliance in the treatment of class II division 1 malocclusion: a geometric morphometric study. Orthodontics \&t craniofacial research, 6(2), pp.88-95.

Teuscher, U., 1978. A growth-related concept for skeletal Class II treatment. American Journal of Orthodontics, 74(3), pp.258-275.

Tümer, N. and Gültan, A.S., 1999. Comparison of the effects of monoblock and twin-block appliances on the skeletal and dentoalveolar structures. American journal of orthodontics and dentofacial orthopedics, 116(4), pp.460-468.

Varlık, S.K., Gültan, A. and Tümer, N., 2008. Comparison of the effects of Twin Block and activator treatment on the soft tissue profile. The European Journal of Orthodontics, 30(2), pp.128-134.

Woodside, D.G., 1973. Some effects of activator treatment on the mandible and the midface. Transactions. European Orthodontic Society, pp.443-447. 